\title{
Role of the 2018 American Thyroid Association statement on postoperative hypoparathyroidism: a 5-year retrospective study
}

Yuxuan Qiu ${ }^{1,2+}$, Zhichao Xing ${ }^{2 \dagger}$, Yuan Fei ${ }^{2}$, Yuanfan Qian ${ }^{2}$, Yan Luo ${ }^{1 *}$ and Anping Su ${ }^{2 *}$

\begin{abstract}
Background: Definitions of postoperative hypoparathyroidism (hypoPT) have never reached consent until the American Thyroid Association (ATA) statement was released, with new characteristics and challenges.

Methods: Patients with papillary thyroid carcinoma who underwent primary total thyroidectomy between January 2013 and June 2018 were retrospectively enrolled. Symptoms of hypocalcemia and their frequency were stringently followed. Patients were divided into groups according to the ATA statement. Incidence of postoperative hypoPT and serum parathyroid hormone levels accompanied by calcium levels, from 1-day to at least 24-month follow-up.

Results: A total of 1749 patients were included: 458 (26.2\%) had transient and 63 (3.6\%) had permanent hypoPT. Transient hypoPT was found in 363 (20.7\%) patients with biochemical hypoPT, 72 (4.1\%) with clinical hypoPT, and 23 (1.3\%) with relative hypoPT; permanent hypoPT was detected in 8 (0.5\%) patients with biochemical hypoPT, 55 (3.1\%) with clinical hypoPT, and none with relative hypoPT. Female sex, age $\geq 55$ years, unintentional parathyroid gland resection, and autotransplantation of $\geq 2$ parathyroid glands were independent risk factors for transient biochemical hypoPT. Age $\geq 55$ years, bilateral central neck dissection, and isthmus tumor location were independent risk factors for transient clinical hypoPT. A postoperative 1-day percentage of parathyroid hormone (PTH) reduction of $>51.1 \%$ was an independent risk factor for relative hypoPT (odds ratio, 4.892; 95\% confidence interval, 1.653-14.480; $P=0.004$ ). No independent risk factor for permanent hypoPT was found.
\end{abstract}

Conclusion: ATA diagnostic criteria for postoperative hypoPT are of great value in differentiating patients by hypocalcemia symptoms and choosing corresponding clinical assistance; however, they may underestimate the actual incidence.

Keywords: Hypoparathyroidism, Hypocalcemia, Thyroidectomy, American Thyroid Association, Risk factors

*Correspondence: luoyan@wchscu.cn; kirkjames@qq.com

${ }^{\dagger}$ Yuxuan Qiu and Zhichao Xing contributed equally to this work

1 Department of Ultrasound, West China Hospital, Sichuan University, No.

37 Guo Xue Xiang, Chengdu 610041, China

${ }^{2}$ Center of Thyroid and Parathyroid Surgery, Sichuan University West

China Hospital, Sichuan Province, No. 37 Guo Xue Xiang, Chengdu, China

\section{Introduction}

In recent years, the global thyroid cancer incidence has risen rapidly, especially for papillary thyroid carcinoma (PTC), which accounts for approximately $85-90 \%$ of all thyroid cancers [1]. Thyroidectomy is the primary treatment for PTC, and postoperative hypoparathyroidism (hypoPT) is one of the most common and serious complications. The main causes of hypoPT are as follows: 
damaged blood supply of the parathyroid gland (PG), which manifests as ischemia or congestion in the PG; direct damage to the PG, including thermal damage or mechanical damage; and accidentally resected PGs. Consequently, insufficient parathyroid hormone (PTH) is produced, and corresponding clinical symptoms are noticed. HypoPT can be divided into transient or permanent hypoPT, and the incidence is approximately $14-60 \%$ and $0-33 \%$, respectively $[2,3]$. The incidence of hypoPT reported in different studies is quite heterogeneous, and the main reasons for this heterogeneity include different surgical procedures, different experience levels of surgeons, and the use of different diagnostic criteria for hypoPT.

The current definition of hypoPT usually involves the following four indicators: (1) clinical manifestations, that is, whether there are symptoms and/or signs of hypocalcemia; (2) biochemical indicators, that is, serum PTH and/or serum calcium levels; (3) treatment indicators, that is, whether calcium and/or vitamin D treatment is needed to maintain no obvious symptoms and/or signs of hypocalcemia; and (4) duration, that is, the cutoff time that distinguishes transient and permanent hypoPT [48]. To solve the problem of complicated and inconsistent diagnostic criteria, the new American Thyroid Association (ATA) statement defines three types of hypoPT: biochemical hypoPT, clinical hypoPT and parathyroid insufficiency (relative hypoPT) [2]. Transient hypoPT is defined as occurring for less than 6 months after surgery, while permanent hypoPT continues beyond 6 months after surgery $[2,9]$. The first two definitions are summaries and supplements to previous literature reports, and there are abundant data and studies for reference. Relative hypoPT requires treatment due to symptoms and/ or signs of hypocalcemia despite the normal range of serum PTH and calcium levels, which is of some clinical significance.

At present, there is no literature to support the risk factors for hypoPT according to the ATA diagnostic criteria. Therefore, the aim of this comparative study grouped by the above three types of hypoPT is to ascertain the incidence and risk factors for each type and to find the predictors of permanent hypoPT.

\section{Methods}

This study is presented in accordance with the STROBE (Strengthening the Reporting of Observational studies in Epidemiology) checklist [10].

\section{Patients}

We performed a retrospective cohort study including all patients with PTC who underwent primary total thyroidectomy with at least unilateral central neck dissection
(CND) at the Center of Thyroid \& Parathyroid Surgery, West China Hospital of Sichuan University from January 2013 to June 2018. The exclusion criteria were severe chronic renal insufficiency, preoperative PG dysfunction, reoperation, endoscopic thyroidectomy, lobectomy, completion thyroidectomy, and less than 2 years of followup. Neoplasms were grouped into stages according to the American Joint Committee for Cancer (AJCC) staging system (8th edition) [11]. The study was approved by the medical ethics committee of West China Hospital, Sichuan University. The patients involved in the study provided informed consent. All methods were performed in accordance with the relevant guidelines and regulations.

\section{Indications of total thyroidectomy with lymph node dissection}

The indications for total thyroidectomy, instead of lobectomy, were as follows: (1) high-risk radiation exposure or a family history of PTC; (2) bilateral or multifocal PTC; (3) unilateral PTC with contralateral thyroid nodule(s); (4) isthmus PTC; (5) stage T3a PTC $>4 \mathrm{~cm}$; (6) PTC with extrathyroidal extension (ETE) (stages T3b and T4); (7) high-risk pathological variants including tall cell variant, diffuse sclerosis variant, and solid variant; (8) PTC with bilateral central neck lymph node or lateral neck lymph node metastases; (9) PTC with distant metastases; and (10) TERT promoter mutation (confirmed by preoperative fine-needle aspiration biopsy). Unilateral CND was performed routinely unless bilateral CND (BCND) was indicated because of (1) bilateral PTC; (2) isthmus PTC; (3) stage T3 and T4 PTC; (4) prelaryngeal and/or pretracheal lymph node metastases; (5) bilateral central lymph node or lateral lymph node metastases; and (6) TERT promoter mutation (confirmed by preoperative fine-needle aspiration biopsy). Therapeutic lateral neck dissection (LND) (preoperative fine-needle aspiration confirmed) was performed in each patient [4].

\section{Surgical procedures}

All surgeries were performed in our center by four surgeons with similar surgical experience levels and styles. Intraoperative neuromonitoring was used to predict postoperative nerve function [12]. At the same time, carbon nanoparticles were recommended for each patient for better identification of PGs, but their use was finally dependent on the patient's will. Generally, thyroid or lymph tissues can be stained black by carbon nanoparticles while parathyroid glands cannot because of their different lymphatic drainage. After the first injection of carbon nanoparticles, every attempt was made to identify and carefully preserve each PG and its blood supply to ensure that at least $1 \mathrm{PG}$ was preserved in situ. The 
resected specimens were examined for any unintentionally removed PGs. When a PG was devascularized or resected unintentionally, selective autotransplantation to the contralateral sternocleidomastoid muscle was performed after confirmation by intraoperative frozen biopsy. The same group of pathologists analyzed all surgical specimens, and the presence of PGs in the surgical specimens was recorded $[4,13]$.

\section{Perioperative management}

Perioperative management of all patients was standardized. Preoperative examinations included serum calcium, PTH, thyroid function, neck ultrasound, and laryngoscopy. Prophylactic calcium supplementation (calcium gluconate $4000 \mathrm{mg}$, intravenous drip) was given to each patient on the same postoperative day. If patients had symptomatic hypocalcemia, added and prolonged oral or intravenous calcium supplementation were administered. Serum PTH and calcium levels were also tested 1 day after surgery.

\section{Follow-up and hypoPT}

Laboratory tests, including tests for serum PTH and calcium levels, were routinely performed at 1 month, 3 months, 6 months, 12 months and 2 years after surgery. If necessary, ${ }^{131}$ I ablation was performed by the Department of Nuclear Medicine 2-3 months after surgery. Postoperative hypoPT was defined according to the ATA statement [2]. Biochemical hypoPT was defined as a low intact PTH level, below the lower limit of our center's standard (1.6-6.9 pmol/L), accompanied by hypocalcemia. Hypocalcemia was defined as a total serum calcium level that was less than the lower limit of our center's standard (2.1-2.7 $\mathrm{mmol} / \mathrm{L})$. Clinical hypoPT was defined as biochemical hypoPT that was accompanied by symptoms and/or signs of hypocalcemia. Relative hypoPT (parathyroid insufficiency) may occur after central neck surgery and typically manifests as clinical symptoms of hypoPT that require medical treatment despite measured laboratory values within normal ranges. Transient hypoPT was defined as occurring for less than 6 months after surgery, while permanent hypoPT continued beyond 6 months after surgery.

\section{Statistical analysis}

All data were analyzed using SPSS 26.0 (SPSS, Chicago, IL, USA) software. Continuous variables (normally distributed) are expressed as mean \pm standard deviation $($ Mean $\pm \mathrm{SD})$; variables not normally distributed were expressed as median (range). The t-test (Student's t-test) and one-factor ANOVA test (analysis of variance) were used for continuous variables that conformed to a normal distribution, and the U-test (Mann-Whitney U-test) was used for those that did not. Pearson Chi-square and Fisher's exact tests were used for categorical variables. Independent risk factors were analyzed using binary logistic regression. $P<0.05$ was considered statistically significant.

\section{Results}

Population characteristics

A total of 1928 patients met the inclusion criteria, of whom 179 (9.3\%) patients withdrew from the follow-up; 1749 patients were finally included in our analysis. Of the 1749 patients, there were 491 (28.1\%) males and 1258 females $(71.9 \%)$. The overall age was $41.52 \pm 11.52$ years old, of whom 217 (12.4\%) patients were aged $\geq 55$ years old. All patients underwent total thyroidectomy, of whom 403 patients (23.0\%) underwent unilateral CND and 1346 (77.0\%) underwent BCND, including 373 (24.3\%) patients who underwent LND. All detailed characteristics are displayed in Table 1. A total of 521 (29.8\%) included patients met the ATA diagnostic criteria: 458 (26.2\%) patients had transient hypoPT, and 63 (3.6\%) patients had permanent hypoPT. Regarding the various types of transient hypoPT, $363(20.7 \%)$ patients had biochemical hypoPT, 72 (4.1\%) had clinical hypoPT, and 23 (1.3\%) had relative hypoPT. Regarding permanent hypoPT, 8 (0.5\%) patients had biochemical hypoPT, 55 (3.1\%) had clinical hypoPT, and no patients had relative hypoPT; 1043 (59.6\%) patients did not have hypoPT. Approximately 185 (10.6\%) patients not only did not meet the ATA diagnostic criteria but were also deemed healthy (e.g., only serum calcium was below the normal range); therefore, they were not included in further data analyses (Fig. 1).

\section{Biochemical hypoPT}

In patients with biochemical hypoPT, 97.8\% (363/371) had transient hypoPT, while only $2.2 \%(8 / 371)$ had permanent hypoPT. In univariate analysis, age $\geq 55$ years $(P=0.016)$, female sex $(P=0.008)$, BCND $(P=0.001)$, PG unintentional resection $(P=0.000)$, and autotransplantation of $\geq 2$ PGs $(P=0.000)$ were associated with transient biochemical hypoPT. In addition, the autotransplantation of $\geq 2$ PGs $(P=0.025)$ was associated with permanent biochemical hypoPT (Table 2).

In multifactor analysis, female sex (odds ratio (OR), 1.411; 95\% confidence interval (CI), 1.067-1.866; $P=0.016)$, age $\geq 55$ years (OR 1.503; 95\% CI 1.046-2.158; $P=0.027$ ), PG unintentional resection (OR 1.703; 95\% CI 1.164-2.491; $P=0.006)$ and autotransplantation of $\geq 2$ PGs (OR 1.634; 95\% CI 1.034-2.580; $P=0.035$ ) were independent risk factors for transient biochemical hypoPT. No independent risk factor for permanent biochemical hypoPT was found. 
Table 1 Population characteristics ( $N=1749)$

\begin{tabular}{|c|c|c|}
\hline Index & $\mathrm{N}$ & $\%$ \\
\hline Age (years)* & $41.52 \pm 11.52$ & - \\
\hline$<55 / \geq 55$ years & $1532 / 217$ & $87.6 / 12.4$ \\
\hline Male/female & $491 / 1258$ & $28.1 / 71.9$ \\
\hline${ }^{131}$ I ablation & 940 & 53.7 \\
\hline $\mathrm{BMI} I^{*}$ & $23.18 \pm 3.91$ & - \\
\hline$<24 / \geq 24$ & $1126 / 623$ & $64.4 / 35.6$ \\
\hline Hypertension & 144 & 8.2 \\
\hline Diabetes & 46 & 2.6 \\
\hline Hypothyroidism & 26 & 1.5 \\
\hline Hyperthyroidism & 47 & 2.7 \\
\hline$H D$ & 387 & 22.1 \\
\hline NG & 908 & 51.9 \\
\hline $\begin{array}{l}\text { Tumor located at } \\
\text { isthmus }\end{array}$ & 85 & 4.9 \\
\hline Bilaterality & 248 & 14.2 \\
\hline Multifocality & 421 & 24.1 \\
\hline Tumor size $(\mathrm{mm})^{*}$ & $13.66 \pm 8.94$ & - \\
\hline Capsular invasion & 366 & 20.9 \\
\hline Minor ETE & 170 & 9.7 \\
\hline Major ETE & 100 & 5.7 \\
\hline UCND/BCND & $403 / 1346$ & 23.0/77.0 \\
\hline ULND/BLND & $328 / 45$ & 18.8/2.6 \\
\hline $\begin{array}{l}\text { Carbon nanopar- } \\
\text { ticles }\end{array}$ & 1287 & 73.6 \\
\hline $\begin{array}{l}\mathrm{Tx} / \mathrm{T} 1 \mathrm{a} / \mathrm{T} 1 \mathrm{~b} / \mathrm{T} 2 / \mathrm{T} 3 \mathrm{a} / \\
\mathrm{T} \mathrm{b} / \mathrm{T} 4 \mathrm{a} / \mathrm{T} 4 \mathrm{~b}\end{array}$ & 10/652/493/118/241/160/73/2 & $\begin{array}{l}0.6 / 37.3 / 28.2 / 6.7 / \\
13.8 / 9.1 / 4.2 / 0.1\end{array}$ \\
\hline $\mathrm{Nx} / \mathrm{N} 0 / \mathrm{N} 1 \mathrm{a} / \mathrm{N} 1 \mathrm{~b}$ & $12 / 857 / 525 / 355$ & $0.7 / 49.0 / 30.0 / 20.3$ \\
\hline Mo/M1 & $1742 / 7$ & $99.6 / 0.4$ \\
\hline
\end{tabular}

BMI, Body mass index; HD, Hashimoto's thyroiditis; NG, Nodular goiter; ETE, extrathyroidal extension; UCND, unilateral central neck dissection; $B C N D$, bilateral central dissection; ULND, unilateral lateral neck dissection; BLND, bilateral lateral neck dissection

*Data presented as mean \pm standard deviation

\section{Clinical hypoPT}

In patients with clinical hypoPT, 56.7\% (72/127) of patients had transient hypoPT, while $43.3 \%(55 / 127)$ had permanent hypoPT, with a ratio of 1.3:1. A higher incidence of transient clinical hypoPT was found in patients aged $\geq 55$ years $(P=0.007)$ and in patients with tumors located at the isthmus $(P=0.001), \mathrm{BCND}$ $(P=0.001), \quad$ and autotransplantation of $\geq 2 \quad$ PGs $(P=0.008)$. Patients with capsular invasion $(P=0.032)$ and intraoperative use of carbon nanoparticles $(P=0.015)$ were less likely to develop transient hypoPT. A lower proportion of permanent clinical hypoPT was found among patients with autotransplantation of $\geq 2$ PGs $(P=0.048)$, whereas a higher proportion of clinical hypoPT was found among those with capsular invasion $(P=0.034)$ (Table 3$)$.

In multifactor analysis, age $\geq 55$ years (OR 2.211; 95\% CI 1.150-4.251; $P=0.017$ ), BCND (OR 2.878; 95\% CI $1.190-6.960 ; P=0.019)$, and tumor location at the isthmus (OR 3.473; 95\% CI 1.601-7.538; $P=0.002$ ) were independent risk factors for transient clinical hypoPT. No independent risk factor for permanent clinical hypoPT was found.

Additionally, patients with clinical hypoPT had a significantly lower PTH level at postoperative day 1 than patients with biochemical hypoPT $(0.83 \pm 0.28 \mathrm{pmol} / \mathrm{L}$ vs $0.93 \pm 0.32 \mathrm{pmol} / \mathrm{L} ; P=0.002$ ).

\section{Relative hypoPT}

Patients with multifocality and bilaterality were more prone to transient relative hypoPT $(P=0.032 ; P=0.014)$. However, in multivariate analysis, no independent risk factors for relative hypoPT were found $(P>0.05$; Table 4$)$. To identify additional predictive factors, receiver operating characteristic (ROC) curve analysis was performed (Fig. 2). ROC analysis showed that the percentage of PTH reduction was of some value, and the best cutoff value was $51.1 \%$, whereas the sensitivity was $82.6 \%$, and the specificity was $50.7 \%$. According to univariate analysis, $82.6 \%(19 / 23)$ of the patients with relative hypoPT had a percentage of $\mathrm{PTH}$ reduction $>51.1 \%$, while the proportion was only $49.2 \%(513 / 1043)$ in the control group, $P=0.002$. Logistic regression confirmed that a percentage reduction of $\mathrm{PTH}>51.1 \%$ at postoperative day 1

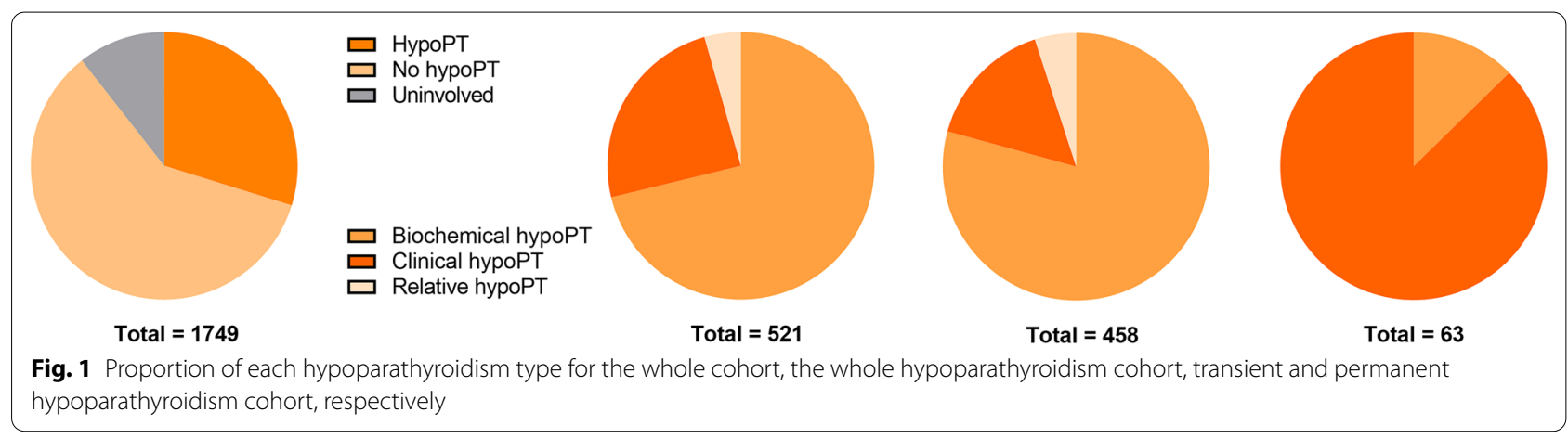


Table 2 Clinical characteristics of Biochemical hypoPT

\begin{tabular}{|c|c|c|c|c|c|}
\hline Index & No hypoPT $(\mathrm{N}=1043)$ & $\begin{array}{l}\text { Transient hypoPT } \\
(\mathrm{N}=363)\end{array}$ & $\begin{array}{l}\text { Permanent hypoPT } \\
(\mathrm{N}=8)\end{array}$ & $P 1$ & $P 2$ \\
\hline Age & $41.06 \pm 11.05$ & $42.67 \pm 12.03$ & $46.25 \pm 7.87$ & $0.019^{*}$ & 0.403 \\
\hline$\geq 55$ years & 109 (10.5) & $55(15.2)$ & $2(25.0)$ & $0.016^{*}$ & 0.355 \\
\hline \multicolumn{6}{|l|}{ Gender } \\
\hline Male & 333 (31.9) & $89(24.5)$ & $1(12.5)$ & $0.008^{*}$ & 0.685 \\
\hline Female & $710(68.1)$ & $274(75.5)$ & $7(87.5)$ & $0.008^{*}$ & 0.685 \\
\hline${ }^{131}$ I & $546(52.3)$ & $205(56.5)$ & $3(37.5)$ & 0.175 & 0.307 \\
\hline BMI & $23.23 \pm 4.12$ & $23.18 \pm 3.84$ & $23.69 \pm 2.51$ & 0.838 & 0.709 \\
\hline$>24$ & $376(36.6)$ & $125(35.2)$ & $4(50.0)$ & 0.645 & 0.462 \\
\hline Hypertension & $84(8.1)$ & $33(9.1)$ & $0(0)$ & 0.538 & 1 \\
\hline Diabetes & $31(3.0)$ & $9(2.5)$ & $0(0)$ & 0.627 & 1 \\
\hline Hypothyroidism & $14(1.3)$ & $6(1.7)$ & $0(0)$ & 0.667 & 1 \\
\hline Hyperthyroidism & $27(2.6)$ & $13(3.6)$ & $0(0)$ & 0.327 & 1 \\
\hline$H D$ & $229(22.0)$ & $90(24.8)$ & $1(12.5)$ & 0.266 & 0.685 \\
\hline NG & $541(51.9)$ & $193(53.2)$ & $7(87.5)$ & 0.670 & 0.074 \\
\hline Pre PTH & $4.96 \pm 1.10$ & $4.95 \pm 1.07$ & $4.00 \pm 1.25$ & 0.878 & $0.014^{*}$ \\
\hline Pre Ca & $2.35 \pm 0.14$ & $2.36 \pm 0.54$ & $2.33 \pm 0.15$ & 0.478 & 0.853 \\
\hline Pre Mg & $0.89 \pm 0.09$ & $0.91 \pm 0.32$ & $0.88 \pm 0.04$ & 0.081 & 0.765 \\
\hline Pre P & $1.12 \pm 0.20$ & $1.22 \pm 1.94$ & $1.13 \pm 0.15$ & 0.099 & 0.895 \\
\hline Pre $25-\mathrm{OH}-\mathrm{VD}$ & $45.81 \pm 17.02$ & $47.91 \pm 14.88$ & $56.28 \pm 12.51$ & 0.275 & 0.338 \\
\hline Carbon nanoparticles & $787(75.5)$ & $259(71.3)$ & $7(87.5)$ & 0.123 & 0.450 \\
\hline Unintentionally resected PGs & $0.09 \pm 0.35$ & $0.19 \pm 0.50$ & 0 & $0.001^{*}$ & $0.000^{*}$ \\
\hline$\geq 1 P G$ & $83(8.0)$ & $52(14.3)$ & $0(0)$ & $0.000^{*}$ & 0.607 \\
\hline Autotransplanted PGs & $0.99 \pm 0.77$ & $1.24 \pm 0.77$ & $0.63 \pm 0.52$ & $0.000^{*}$ & $0.025^{*}$ \\
\hline NoPG & $298(28.6)$ & $66(18.2)$ & $3(37.5)$ & $0.000^{*}$ & 0.171 \\
\hline $1 P G$ & $474(45.4)$ & $151(41.6)$ & $5(62.5)$ & 0.220 & 0.289 \\
\hline$\geq 2 P G s$ & $271(26.0)$ & $146(40.2)$ & $0(0)$ & $0.000^{*}$ & $0.025^{*}$ \\
\hline \multicolumn{6}{|l|}{ Neck dissection } \\
\hline UCND & $277(26.6)$ & $65(17.9)$ & $3(37.5)$ & $0.001^{*}$ & 0.165 \\
\hline BCND & $766(73.4)$ & $298(82.1)$ & $5(62.5)$ & $0.001^{*}$ & 0.165 \\
\hline ULND & $184(17.6)$ & $74(20.4)$ & $1(12.5)$ & 0.245 & 1 \\
\hline BLND & $21(2.0)$ & $14(3.9)$ & $0(0)$ & 0.052 & 1 \\
\hline Intraoperative blood loss (mL) & $44.25 \pm 41.11$ & $58.11 \pm 63.77$ & $17.00 \pm 4.47$ & $0.000^{*}$ & 0.151 \\
\hline Intraoperative liquid infusion ( $\mathrm{mL})$ & $1330 \pm 700$ & $1500 \pm 685$ & $1208 \pm 311$ & $0.000^{*}$ & 0.299 \\
\hline \multicolumn{6}{|l|}{ Tumor characteristics } \\
\hline Isthmus & $48(4.8)$ & $16(4.5)$ & $0(0)$ & 0.826 & 1 \\
\hline Multifocality & $142(14.1)$ & $52(14.6)$ & $1(12.5)$ & 0.830 & 1 \\
\hline Bilaterality & $245(24.0)$ & $80(22.2)$ & $1(12.5)$ & 0.516 & 1 \\
\hline Capsular invasion & $227(21.8)$ & $66(18.2)$ & $2(25.0)$ & 0.148 & 0.642 \\
\hline Minor ETE & $95(9.1)$ & $44(12.1)$ & $1(12.5)$ & 0.098 & 1 \\
\hline Major ETE & $56(5.4)$ & $26(7.2)$ & $2(25.0)$ & 0.209 & 0.116 \\
\hline
\end{tabular}

BMI, Body mass index; HD, Hashimoto's thyroiditis; NG, Nodular goiter; Pre, preoperative; PTH, Parathyroid Hormone; Ca, calcium; Mg, Magnesium; P, phosphorus; PG, Parathyroid Gland; UCND, unilateral central neck dissection; BCND, bilateral central dissection; ULND, unilateral lateral neck dissection; BLND, bilateral lateral neck dissection; ETE, extrathyroidal extension

${ }^{*} P<0.05$

significantly increased the risk of relative hypoPT (OR 4.892; 95\% CI 1.653-14.480; $P=0.004$ ).
Discussion

Many definitions and diagnostic criteria of hypoPT after thyroid surgery are found in the existing literature, leading to widespread controversy over its true 
Table 3 Clinical characteristics of Clinical hypoPT

\begin{tabular}{|c|c|c|c|c|c|}
\hline Index & No hypoPT ( $N=1043)$ & $\begin{array}{l}\text { Transient hypoPT } \\
(\mathrm{N}=72)\end{array}$ & $\begin{array}{l}\text { Permanent hypoPT } \\
(\mathrm{N}=55)\end{array}$ & $P 1$ & $P 2$ \\
\hline Age & $41.06 \pm 11.05$ & $41.88 \pm 13.45$ & $40.89 \pm 13.37$ & 0.615 & 0.683 \\
\hline$\geq 55$ years & $109(10.5)$ & $15(20.8)$ & $9(16.4)$ & $0.007^{*}$ & 0.524 \\
\hline \multicolumn{6}{|l|}{ Gender } \\
\hline Male & 333 (31.9) & $16(22.2)$ & $13(23.6)$ & 0.086 & 0.851 \\
\hline Female & $710(68.1)$ & $56(77.8)$ & $42(76.4)$ & 0.086 & 0.851 \\
\hline${ }^{131} \mid$ & $546(52.3)$ & $38(52.8)$ & $36(65.5)$ & 0.944 & 0.151 \\
\hline BMI & $23.23 \pm 4.12$ & $23.29 \pm 3.06$ & $23.07 \pm 3.88$ & 0.910 & 0.728 \\
\hline$>24$ & $376(36.6)$ & $29(42.0)$ & $18(32.7)$ & 0.364 & 0.289 \\
\hline Hypertension & $84(8.1)$ & $6(8.3)$ & $3(5.5)$ & 0.933 & 0.731 \\
\hline Diabetes & $31(3.0)$ & $2(2.8)$ & $2(3.6)$ & 1 & 1 \\
\hline Hypothyroidism & $14(1.3)$ & $1(1.4)$ & $1(1.8)$ & 1 & 1 \\
\hline Hyperthyroidism & $27(2.6)$ & $1(1.4)$ & $0(0)$ & 1 & 1 \\
\hline $\mathrm{HD}$ & $229(22.0)$ & $12(16.7)$ & $15(27.3)$ & 0.292 & 0.148 \\
\hline NG & $541(51.9)$ & $31(43.1)$ & $30(54.5)$ & 0.148 & 0.199 \\
\hline Pre PTH & $4.96 \pm 1.10$ & $4.91 \pm 1.19$ & $4.82 \pm 1.10$ & 0.698 & 0.673 \\
\hline Pre Ca & $2.35 \pm 0.14$ & $2.32 \pm 0.10$ & $2.32 \pm 0.10$ & $0.042^{*}$ & 0.925 \\
\hline Pre Mg & $0.89 \pm 0.09$ & $0.91 \pm 0.18$ & $0.88 \pm 0.08$ & 0.192 & 0.305 \\
\hline Pre P & $1.12 \pm 0.20$ & $1.12 \pm 0.20$ & $1.14 \pm 0.17$ & 0.893 & 0.508 \\
\hline Pre 25-OH-VD & $45.81 \pm 17.02$ & $46.79 \pm 16.08$ & $48.53 \pm 27.66$ & 0.759 & 0.788 \\
\hline Carbon nanoparticles & $787(75.5)$ & $45(62.5)$ & $41(74.5)$ & $0.015^{*}$ & 0.150 \\
\hline Unintentionally resected PGs & $0.09 \pm 0.35$ & $0.08 \pm 0.28$ & $0.13 \pm 0.34$ & 0.799 & 0.422 \\
\hline$\geq 1 P G$ & $83(8.0)$ & $6(8.3)$ & $7(12.7)$ & 0.909 & 0.418 \\
\hline Autotransplanted PGs & $0.99 \pm 0.77$ & $1.29 \pm 0.70$ & $1.13 \pm 0.70$ & $0.001^{*}$ & 0.191 \\
\hline No PG & $298(28.6)$ & $9(12.5)$ & $8(14.5)$ & $0.003^{*}$ & 0.737 \\
\hline $1 P G$ & $474(45.4)$ & $34(47.2)$ & $34(61.8)$ & 0.770 & 0.102 \\
\hline$\geq 2 P G s$ & $271(26.0)$ & $29(40.3)$ & $13(23.6)$ & $0.008^{*}$ & $0.048^{*}$ \\
\hline \multicolumn{6}{|l|}{ Neck dissection } \\
\hline UCND & $277(26.6)$ & $6(8.3)$ & $7(12.7)$ & $0.001^{*}$ & 0.418 \\
\hline $\mathrm{BCND}$ & $766(73.4)$ & $66(91.7)$ & $48(87.3)$ & $0.001^{*}$ & 0.418 \\
\hline ULND & $184(17.6)$ & $16(22.2)$ & $14(25.5)$ & 0.327 & 0.671 \\
\hline BLND & $21(2.0)$ & $4(5.6)$ & $2(3.6)$ & 0.072 & 0.697 \\
\hline Intraoperative blood loss (mL) & $44.25 \pm 41.11$ & $69.49 \pm 111.98$ & $62.10 \pm 87.73$ & 0.069 & 0.699 \\
\hline Intraoperative liquid infusion (mL) & $1330 \pm 700$ & $1526 \pm 705$ & $1678 \pm 829$ & $0.028^{*}$ & 0.295 \\
\hline \multicolumn{6}{|l|}{ Tumor characteristics } \\
\hline Isthmus & $48(4.8)$ & $10(14.3)$ & $2(3.6)$ & $0.001^{*}$ & 0.065 \\
\hline Multifocality & $142(14.1)$ & $20(28.2)$ & $18(32.7)$ & 0.430 & 0.696 \\
\hline Bilaterality & $245(24)$ & $11(15.7)$ & $13(23.6)$ & 0.716 & 0.264 \\
\hline Capsular invasion & $227(21.8)$ & $8(11.1)$ & $14(25.5)$ & $0.032^{*}$ & $0.034^{*}$ \\
\hline Minor ETE & $95(9.1)$ & $7(55.6)$ & $3(5.5)$ & 0.863 & 0.512 \\
\hline Major ETE & $56(5.4)$ & $4(9.7)$ & $4(7.2)$ & 0.792 & 0.726 \\
\hline
\end{tabular}

BMI, Body mass index; HD, Hashimoto's thyroiditis; NG, Nodular goiter; Pre, preoperative; PTH, Parathyroid Hormone; Ca, calcium; Mg, Magnesium; P, phosphorus; PG, Parathyroid Gland; UCND, unilateral central neck dissection; BCND, bilateral central dissection; ULND, unilateral lateral neck dissection; BLND, bilateral lateral neck dissection; ETE, extrathyroidal extension

${ }^{*} P<0.05$

incidence. According to a study by Mehanna et al. in 2010, the definition of postoperative hypoPT is almost the most important factor affecting its incidence [14]. The study used 10 different definitions of permanent hypoPT and found that its incidence varied from 0.9 to $4.4 \%$ [14]. Furthermore, as postoperative hypoPT began 
Table 4 Clinical characteristics of relative hypoPT

\begin{tabular}{|c|c|c|c|}
\hline Index & No hypoPT (N=1043) & Transient hypoPT $(\mathrm{N}=23)$ & $P$ \\
\hline Age & $41.06 \pm 11.05$ & $45.22 \pm 9.08$ & 0.073 \\
\hline$\geq 55$ years & $109(10.5)$ & $3(13.0)$ & 0.726 \\
\hline \multicolumn{4}{|l|}{ Gender } \\
\hline Male & $333(31.9)$ & $6(26.1)$ & 0.552 \\
\hline Female & $710(68.1)$ & $17(73.9)$ & 0.552 \\
\hline${ }^{131}$ & $546(52.3)$ & $16(69.6)$ & 0.102 \\
\hline BMI & $23.23 \pm 4.12$ & $24.34 \pm 2.81$ & 0.207 \\
\hline$>24$ & $376(36.6)$ & $12(54.5)$ & 0.084 \\
\hline Hypertension & $84(8.1)$ & $3(13.0)$ & 0.426 \\
\hline Diabetes & $31(3.0)$ & $0(0)$ & 1 \\
\hline Hypothyroidism & $14(1.3)$ & $1(4.3)$ & 0.281 \\
\hline Hyperthyroidism & $27(2.6)$ & $0(0)$ & 1 \\
\hline $\mathrm{HD}$ & $229(22)$ & $2(8.7)$ & 0.197 \\
\hline NG & $541(51.9)$ & $14(60.9)$ & 0.393 \\
\hline Pre PTH & $4.96 \pm 1.1$ & $5.49 \pm 1.03$ & $0.020^{*}$ \\
\hline Pre Ca & $2.35 \pm 0.14$ & $2.33 \pm 0.08$ & 0.610 \\
\hline Pre Mg & $0.89 \pm 0.09$ & $0.95 \pm 0.09$ & $0.004^{*}$ \\
\hline Pre P & $1.12 \pm 0.20$ & $1.07 \pm 0.15$ & 0.256 \\
\hline Pre 25-OH-VD & $45.81 \pm 17.02$ & $50.00 \pm 15.40$ & 0.467 \\
\hline Carbon nanoparticles & $787(75.5)$ & $18(78.3)$ & 1 \\
\hline Unintentionally resected PGs & $0.09 \pm 0.35$ & $0.04 \pm 0.21$ & 0.486 \\
\hline$\geq 1 P G$ & $83(8.0)$ & $1(4.3)$ & 1 \\
\hline Autotransplanted PGs & $0.99 \pm 0.77$ & $1.26 \pm 0.75$ & 0.092 \\
\hline NoPG & $298(28.6)$ & $3(13.0)$ & 0.157 \\
\hline $1 P G$ & $474(45.4)$ & $12(52.2)$ & 0.522 \\
\hline$\geq 2 \mathrm{PGs}$ & $271(26.0)$ & $8(34.8)$ & 0.342 \\
\hline \multicolumn{4}{|l|}{ Neck dissection } \\
\hline UCND & $277(26.6)$ & $8(34.8)$ & 0.378 \\
\hline BCND & $766(73.4)$ & $15(65.2)$ & 0.378 \\
\hline ULND & $184(17.6)$ & $7(30.4)$ & 0.114 \\
\hline BLND & $21(2.0)$ & $0(0)$ & 1 \\
\hline Intraoperative blood loss (mL) & $44.25 \pm 41.11$ & $57.83 \pm 63.17$ & 0.316 \\
\hline Intraoperative liquid infusion (mL) & $1330 \pm 700$ & $1434 \pm 895$ & 0.494 \\
\hline \multicolumn{4}{|l|}{ Tumor characteristics } \\
\hline Isthmus & $48(4.8)$ & $0(0)$ & 0.619 \\
\hline Multifocality & $142(14.1)$ & $10(43.5)$ & $0.032^{*}$ \\
\hline Bilaterality & $245(24)$ & $7(33.3)$ & $0.014^{*}$ \\
\hline Capsular invasion & $227(21.8)$ & $2(8.7)$ & 0.197 \\
\hline Minor ETE & $95(9.1)$ & $4(17.4)$ & 0.262 \\
\hline Major ETE & $56(5.4)$ & $2(8.7)$ & 0.360 \\
\hline
\end{tabular}

BMI, Body mass index; HD, Hashimoto's thyroiditis; NG, Nodular goiter; Pre, preoperative; PTH, Parathyroid Hormone; Ca, calcium; Mg, Magnesium; P, phosphorus; PG, Parathyroid Gland; UCND, unilateral central neck dissection; BCND, bilateral central dissection; ULND, unilateral lateral neck dissection; BLND, bilateral lateral neck dissection; ETE, extrathyroidal extension

${ }^{*} P<0.05$

to be noticed, clinicians began an attempt to standardize the diagnostic criteria for postoperative hypoPT. According to a recent systematic review, researchers in $41.6 \%$ of studies used hypocalcemia as the definition of permanent hypoPT, and the corresponding incidence was $1.63 \%$ (95\% CI $0.98-2.42 \%$ ); researchers in $22.5 \%$ of studies used the lower limit of normal serum calcium range as the criterion for permanent hypoPT, 


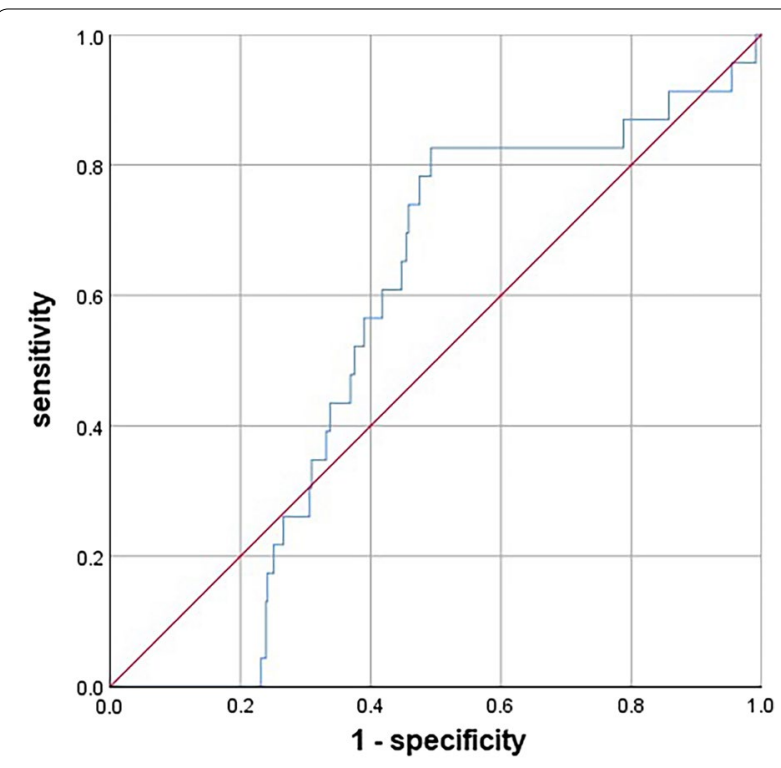

Fig. 2 Receiver operating characteristic (ROC) curve for decline rate of parathyroid hormone levels at postoperative 1-day for predicting relative hypothyroidism

and the corresponding incidence was $2.71 \%$ (95\% CI $1.48-4.37 \%$ ); and researchers in $36.0 \%$ of studies did not describe their definition of permanent hypoPT, and the incidence was $2.97 \%$ (95\% CI $1.95-4.26 \%$ ) [6]. As the first study following the ATA statement, we found that the overall incidence of hypoPT was $29.8 \%$, of which the transient hypoPT incidence was $26.2 \%$ and the permanent hypoPT incidence was $3.6 \%$. The incidence of postoperative biochemical hypoPT was $21.2 \%$, including $20.7 \%$ transient hypoPT and $0.5 \%$ permanent hypoPT. The incidence of clinical hypoPT was $7.2 \%$, including $4.1 \%$ transient hypoPT, $3.1 \%$ permanent hypoPT, and $1.3 \%$ relative hypoPT, all of which were transient.

In this study, female sex was the main independent risk factor for postoperative hypoPT. In a study involving 903 patients, 399 (44.4\%) of whom were diagnosed with hypoPT, female sex was an independent risk factor for transient hypoPT (OR 1.818; 95\% CI 1.326-2.491; $P<0.001)$ but not for permanent hypoPT $(P=0.512)$ [15]. A meta-analysis including 10 studies with 3860 patients who underwent at least total thyroidectomy found that the incidence of postoperative transient hypocalcemia in female patients was significantly higher than that in male patients (OR 1.70; 95\% CI 1.03-2.80) [16]. However, the mechanism behind this risk factor for females is not yet clear $[7,17,18]$. There might be differences in the effect of sex hormones on PTH secretion [19]. Different sexes may have different parathyroid tissue monoclonal proliferation and mitotic regulators [20, 21]. Furthermore, there may be differences in the anatomy and morphology of PGs between male and female patients [22, 23]. In addition, studies have shown that this sex difference may be due to the higher rate of vitamin D deficiency in female patients [24].

Patients who underwent total thyroidectomy with CND had a significantly higher incidence of postoperative hypoPT than patients who did not undergo CND. Chisholm et al's meta-analysis proved this significantly increased risk, with a risk difference of 7.7 (95\% CI 5.614.3) [25]. At present, the main issue is whether prophylactic CND should be implemented. Docimo et al. reported symptomatic hypocalcemia developed only in $6.3 \%$ of patients, by adopting total thyroidectomy without prophylactic central lymphadenectomy [26]. A large meta-analysis of 23 cohort studies showed that prophylactic CND significantly reduced local recurrence of PTC (OR 0.65; 95\% CI 0.48-0.88) but increased postoperative transient hoarseness (OR 2.03; 95\% CI 1.32-3.13), transient hypocalcemia (OR 2.23; 95\% CI 1.84-2.70) and permanent hypocalcemia (OR 2.22; 95\% CI 1.58-3.13) [27]. Compared with unilateral CND, BCND can significantly increase the risk of recurrent laryngeal nerve injury and postoperative hypoPT, but the risk of local recurrence is not improved [28, 29]. Currently, there is agreement between endocrine and neck surgeons about the extension of therapeutic lymph node dissection in $\mathrm{N}+\mathrm{PTC}$ patients and in the prophylactic treatment of No "high risk" patients [30].

In this study, the autotransplantation of only 1 PG had no significant effect on the incidence of transient or permanent hypoPT, while the autotransplantation of $\geq 2$ PGs increased the risk for transient hypoPT, but the incidence of permanent hypoPT did not change significantly, even though a preventive trend was detected. Su et al. studied how the number of autotransplanted PGs affected the incidence of postoperative hypoPT. Among the 766 enrolled patients, $36.9 \%$ (283 patients) did not undergo PG autotransplantation, and 48.7\% (373 patients), 12.7\% (97 patients) and 1.7\% (13 patients) underwent the autotransplantation of 1,2 , and 3 PGs, respectively. With an increase in the number of autotransplanted PGs of 0 , 1,2 , and 3 PGs, the incidence of transient hypoPT was $26.1 \%, 36.2 \%, 52.6 \%$, and $84.6 \%$, respectively $(P<0.05)$, and the incidence of permanent hypoPT was $1.8 \%, 1.1 \%$, $1.0 \%$ and $0 \%$, respectively $(P>0.05)$ [4]. Most studies support that intraoperative parathyroid autotransplantation will significantly increase the incidence of transient hypoPT but can effectively decrease the risk of permanent hypoPT. A meta-analysis of 25 original studies found that the autotransplantation of 1 or 2-3 PGs increased the risk of postoperative transient hypoPT (OR 1.71; 95\% CI 1.25-2.35; $P=0.001$; OR 2.22; 95\% CI 
$1.43-3.45 ; P<0.001)$, but there was no significant difference in the risk of hypoPT at 6 months after surgery (OR 1.09; 95\% CI 0.59-2.01; $P=0.781$; OR 0.55 ; 95\% CI $0.16-1.87 ; P=0.341$ ) [16]. Therefore, it is recommended to first emphasize the preservation of both upper PGs in situ and strategic or selective autotransplantation of lower PGs.

The ATA statement chose 6 months as the cutoff time between transient and permanent hypoPT. Our study found that PTH levels recovered between 6 and 12 months after surgery in $16(25.4 \%)$ patients diagnosed with permanent hypoPT, and PTH levels recovered or did not recover after 12 months in 47 (74.6\%) patients. From this result, the recovery of parathyroid function within 6 to 12 months is still expected. El-Sharaky et al. believe that most autotransplanted PGs need at least 2-6 weeks to gradually recover their function, but some autotransplanted PGs can take several months to recover [31]. If the blood supply of the PGs preserved in situ is damaged during the operation, the central part of the PGs is affected by avascular necrosis and the formation of new blood vessels for a long time, and functional recovery will be slower [32]. In a study of 854 patients undergoing total thyroidectomy, Villarroya et al. found that a total of $14.5 \%$ (142 patients) had postoperative hypoPT, in whom parathyroid function recovered within 6 months in $8.5 \%$ (73 patients), recovered within 6-12 months in $2.5 \%$ (21 patients), recovered within more than 1 year in $1.4 \%$ (12 patients), and did not recover during follow-up in $4.2 \%$ (36 patients) [33]. Kim et al. enrolled 1467 patients with total thyroidectomy, with $1.5 \%$ (22 patients) diagnosed with permanent hypoPT based on the 6-month time point, but they found that parathyroid function recovered in $0.3 \%$ ( 5 patients) after 6 months [32]. Ritter et al. followed up 1054 patients who underwent total thyroidectomy. A total of $17.9 \%$ (189 patients) had hypoPT after surgery, of whom parathyroid function recovered within 2 months in 12.5\% (132 patients), recovered between 6 and 12 months in $0.9 \%$ ( 9 patients), and did not recover after 12 months in only $1.9 \%$ (20 patients) [34].

In addition to the probable limitation regarding cutoff time, the severity of accompanying hypocalcemia should be taken into consideration. Therefore, we propose the following suggestions about the diagnostic criteria of hypoPT: (1) patients with hypocalcemia (serum calcium level below the lower limit of normal range) needing calcium and/or vitamin D supplementation to relieve the symptoms of hypocalcemia and/or (2) any patient with a serum PTH level lower than the lower limit of normal range. In addition, the cutoff time to distinguish transient and permanent hypoPT should be 1 year. HypoPT should also be divided into mild, moderate, and severe types: mild is defined as hypoPT in which the serum PTH level lower than the lower limit of the normal reference value, with no symptoms of hypocalcemia and no need for calcium and/or vitamin D supplementation (except those who need supplementation due to vitamin $\mathrm{D}$ deficiency); moderate is defined as hypoPT in which only oral calcium and/or vitamin D supplementation can relieve the symptoms of hypocalcemia; and severe is defined as hypoPT in which intravenous injection of calcium and/ or vitamin D can alleviate the symptoms of hypocalcemia or need of injectable rhPTH (1-84) and other longacting PTH analogs to keep asymptomatic. In addition, the effects of mental and psychological factors (such as hysteria) should be excluded for moderate and severe cases. These suggestions fully take the impact of symptoms of hypocalcemia on the quality of life of patients into account. Transient symptoms of hypocalcemia that can be relieved by oral calcium supplementation will hardly affect the quality of life of patients. Patients with severe hypoPT need intravenous calcium supplementation to maintain no symptoms of hypocalcemia; therefore, the quality of life of these patients will be greatly affected by the more serious symptoms of hypocalcemia and the necessity to go to a medical institution for injection treatment.

Our study is subject to its retrospective nature and withdrawal rate $(9.3 \%)$. In addition, there might be some subjective bias of the symptoms of hypocalcemia during follow-up.

\section{Conclusion}

The ATA diagnostic criteria for postoperative hypoPT are of great value in differentiating patients by hypocalcemia symptoms and choosing corresponding clinical assistance, though they may underestimate the actual incidence.

\section{Abbreviations}

hypoPT: Hypoparathyroidism; ATA: American Thyroid Association; PTH: Parathyroid hormone; PTC: Papillary thyroid carcinoma; PG: Parathyroid gland; CND: Central neck dissection; ETE: Extrathyroidal extension; BCND: Bilateral central neck dissection.

\section{Acknowledgements}

The authors thank the entire staff of the participating departments for their expert assistance.

\section{Authors' contributions}

$Y Q$ and ZX conceived and designed the study. YQ, ZX, YF and YQ executed the study. YQ, YF and $Y L$ analyzed and involved in interpretation of data. $Y Q, Z X$ and $A S$ drafted the article. YQ, YL and AS made final approval of the version to be published. All authors read and approved the final manuscript.

Funding

This work was supported by the 1.3.5 project for disciplines of excellence, West China Hospital, Sichuan University (ZY2017309). 


\section{Availability of data and materials}

The data that support the findings of this study are available from the corresponding author upon reasonable request.

\section{Declarations}

Ethics approval and consent to participate

This study was in accordance with the Declaration of Helsinki and has been approved by the ethics committee of West China Hospital, Sichuan University.

\section{Consent for publication}

Not applicable.

\section{Competing interests}

The authors declared that they have no conflicts of interest in this work.

Received: 24 February 2021 Accepted: 28 August 2021

Published online: 02 September 2021

\section{References}

1. Qiu Y, Fei Y, Liu J, Liu C, He X, Zhu N, Zhao WJ, Zhu JQ. Prevalence, risk factors and location of skip metastasis in papillary thyroid carcinoma: a systematic review and meta-analysis. Cancer Manag Res. 2019;11:8721-30.

2. Orloff LA, Wiseman SM, Bernet VJ, Fahey TJ 3rd, Shaha AR, Shindo ML, Snyder SK, Stack BC Jr, Sunwoo JB, Wang MB. American Thyroid Association statement on postoperative hypoparathyroidism: diagnosis, prevention, and management in adults. Thyroid. 2018;28(7):830-41.

3. Haugen BR, Alexander EK, Bible KC, Doherty GM, Mandel SJ, Nikiforov YE, Pacini F, Randolph GW, Sawka AM, Schlumberger M, et al. 2015 American Thyroid Association management guidelines for adult patients with thyroid nodules and differentiated thyroid cancer: the american thyroid association guidelines task force on thyroid nodules and differentiated thyroid cancer. Thyroid. 2016;26(1):1-133.

4. Su A, Gong Y, Wu W, Gong R, Li Z, Zhu J. Does the number of parathyroid glands autotransplanted affect the incidence of hypoparathyroidism and recovery of parathyroid function? Surgery. 2018;164:124-9.

5. Ponce de Leon-Ballesteros G, Velazquez-Fernandez D, HernandezCalderon FJ, Bonilla-Ramirez C, Perez-Soto RH, Pantoja JP, Sierra M, Herrera MF. Hypoparathyroidism after total thyroidectomy: importance of the intraoperative management of the parathyroid glands. World I Surg. 2019;43(7):1728-35.

6. Harsløf T, Rolighed L, Rejnmark L. Huge variations in definition and reported incidence of postsurgical hypoparathyroidism: a systematic review. Endocrine. 2019;64(1):176-83.

7. Díez JJ, Anda E, Sastre J, Pérez Corral B, Álvarez-Escolá C, Manjón L, Paja M, Sambo M, Santiago Fernández P, Blanco Carrera C, et al. Prevalence and risk factors for hypoparathyroidism following total thyroidectomy in Spain: a multicentric and nation-wide retrospective analysis. Endocrine. 2019;66(2):405-15.

8. Teshima M, Otsuki N, Morita N, Furukawa T, Shinomiya H, Shinomiya H, Nibu KI. Postoperative hypoparathyroidism after total thyroidectomy for thyroid cancer. Auris Nasus Larynx. 2018;45(6):1233-8.

9. Shoback DM, Bilezikian JP, Costa AG, Dempster D, Dralle H, Khan AA, Peacock M, Raffaelli M, Silva BC, Thakker RV, et al. Presentation of hypoparathyroidism: etiologies and clinical features. J Clin Endocrinol Metab. 2016;101(6):2300-12.

10. Little J, Higgins JPT, loannidis JPA, Moher D, Gagnon F, von Elm E, Khoury MJ, Cohen B, Davey-Smith G, Grimshaw J, et al. STrengthening the REporting of Genetic Association studies (STREGA) - an extension of the STROBE statement. Eur J Clin Invest. 2009;39(4):247-66.

11. Zanoni DK, Patel SG, Shah JP. Changes in the 8th edition of the American Joint Committee on Cancer (AJCC) staging of head and neck cancer: rationale and implications. Curr Oncol Rep. 2019;21(6):52.

12. Calò PG, Medas F, Conzo G, Podda F, Canu GL, Gambardella C, Pisano G, Erdas E, Nicolosi A. Intraoperative neuromonitoring in thyroid surgery: is the two-staged thyroidectomy justified? Int I Surg. 2017:41 (Suppl 1):S13-20.
13. Xing Z, Qiu Y, Xia B, Abuduwaili M, Fei Y, Zhu J, Su A. Surgical strategy when identifying less than four parathyroid glands during total thyroidectomy: a retrospective cohort study. Gland Surg. 2021;10(1):10-22.

14. Mehanna HM, Jain A, Randeva H, Watkinson J, Shaha A. Postoperative hypocalcemia-the difference a definition makes. Head Neck. 2010;32(3):279-83.

15. Su A, Wang B, Gong Y, Gong R, Li Z, Zhu J. Risk factors of hypoparathyroidism following total thyroidectomy with central lymph node dissection. Medicine. 2017;96(39):e8162.

16. Wang B, Zhu C-R, Liu H, Wu J. The effectiveness of parathyroid gland autotransplantation in preserving parathyroid function during thyroid surgery for thyroid neoplasms: a meta-analysis. PLOS ONE. 2019;14(8):e0221173.

17. Cho JN, Park WS, Min SY. Predictors and risk factors of hypoparathyroidism after total thyroidectomy. Int J Surg. 2016;34:47-52.

18. Sands NB, Payne RJ, Côté V, Hier MP, Black MJ, Tamilia M. Female gender as a risk factor for transient post-thyroidectomy hypocalcemia. Otolaryngol Head Neck Surg. 2011;145(4):561-4.

19. Sandelin K, Skoog L, Humla S, Farnebo LO. Oestrogen, progesterone, and glucocorticoid receptors in normal and neoplastic parathyroid glands. Eur J Surg. 1992;158(9):467-72.

20. Almaden Y, Felsenfeld AJ, Rodriguez M, Cañadillas S, Luque F, Bas A, Bravo J, Torregrosa V, Palma A, Ramos B, et al. Proliferation in hyperplastic human and normal rat parathyroid glands: role of phosphate, calcitriol, and gender. Kidney Int. 2003;64(6):2311-7.

21. Naveh-Many T, Rahamimov R, Livni N, Silver J. Parathyroid cell proliferation in normal and chronic renal failure rats. The effects of calcium, phosphate, and vitamin D. J Clin Investig. 1995;96(4):1786-93.

22. Dufour DR, Wilkerson SY. Factors related to parathyroid weight in normal persons. Arch Pathol Lab Med. 1983;107(4):167-72.

23. Dekker A, Dunsford HA, Geyer SJ. The normal parathyroid gland at autopsy: the significance of stromal fat in adult patients. J Pathol. 1979;128(3):127-32.

24. Erbil Y, Barbaros U, Temel B, Turkoglu U, Işsever H, Bozbora A, Ozarmağan S, Tezelman S. The impact of age, vitamin D(3) level, and incidental parathyroidectomy on postoperative hypocalcemia after total or near total thyroidectomy. Am J Surg. 2009;197(4):439-46.

25. Chisholm EJ, Kulinskaya E, Tolley NS. Systematic review and meta-analysis of the adverse effects of thyroidectomy combined with central neck dissection as compared with thyroidectomy alone. Laryngoscope. 2009;119(6):1135-9.

26. Docimo G, Tolone S, Ruggiero R, Gubitosi A, Pasquali D, De Bellis A, Limongelli P, Del Genio G, Docimo L, Conzo G. Total thyroidectomy without prophylactic central neck dissection combined with routine oral calcium and vitamin D supplements: is it a good option to achieve a low recurrence rate avoiding hypocalcemia? A retrospective study. Miner Chir. 2013;68(3):321-8.

27. Chen L, Wu Y-H, Lee C-H, Chen H-A, Loh E-W, Tam K-W. Prophylactic central neck dissection for papillary thyroid carcinoma with clinically uninvolved central neck lymph nodes: a systematic review and metaanalysis. World J Surg. 2018;42(9):2846-57.

28. Roh J-L, Park J-Y, Park Cl. Total thyroidectomy plus neck dissection in differentiated papillary thyroid carcinoma patients: pattern of nodal metastasis, morbidity, recurrence, and postoperative levels of serum parathyroid hormone. Ann Surg. 2007;245(4):604-10.

29. Lee YS, Kim SW, Kim SW, Kim SK, Kang H-S, Lee ES, Chung K-W. Extent of routine central lymph node dissection with small papillary thyroid carcinoma. World J Surg. 2007;31(10):1954-9.

30. Conzo G, Docimo G, Mauriello C, Gambardella C, Esposito D, Cavallo F, Tartaglia E, Napolitano S, Santini L. The current status of lymph node dissection in the treatment of papillary thyroid cancer. A literature review. Clin Ter. 2013;164(4):e343-6.

31. El-Sharaky MI, Kahalil MR, Sharaky O, Sakr MF, Fadaly GA, El-Hammadi HA, Moussa MM. Assessment of parathyroid autotransplantation for preservation of parathyroid function after total thyroidectomy. Head Neck. 2003;25(10):799-807.

32. Kim S-M, Kim HK, Kim K-J, Chang HJ, Kim B-W, Lee YS, Chang H-S, Park CS. Recovery from permanent hypoparathyroidism after total thyroidectomy. Thyroid. 2015;25(7):830-3.

33. Villarroya-Marquina I, Sancho J, Lorente-Poch L, Gallego-Otaegui L, Sitges-Serra A. Time to parathyroid function recovery in patients with 
protracted hypoparathyroidism after total thyroidectomy. Eur J Endocrinol. 2018;178(1):103-11.

34. Ritter K, Elfenbein D, Schneider DF, Chen H, Sippel RS. Hypoparathyroidism after total thyroidectomy: incidence and resolution. J Surg Res. 2015;197(2):348-53.

\section{Publisher's Note}

Springer Nature remains neutral with regard to jurisdictional claims in published maps and institutional affiliations.
Ready to submit your research? Choose BMC and benefit from:

- fast, convenient online submission

- thorough peer review by experienced researchers in your field

- rapid publication on acceptance

- support for research data, including large and complex data types

- gold Open Access which fosters wider collaboration and increased citations

- maximum visibility for your research: over $100 \mathrm{M}$ website views per year

At BMC, research is always in progress.

Learn more biomedcentral.com/submissions 